\title{
Frequency of computed tomography abnormalities in patients with chronic thromboembolic pulmonary hypertension: a comparative study between lung perfusion scan and computed tomography pulmonary angiography
}

\author{
Ahmed Fathala, ${ }^{1}$ Alaa Aldurabi ${ }^{2}$ \\ ${ }^{1}$ Department of Radiology, Nuclear Medicine and Cardiovascular Imaging, King Faisal Specialist Hospital and Research \\ Center, Riyadh \\ ${ }^{2}$ Department of Radiology, College of Medicine, Qassim University, Buraidah, Saudi Arabia
}

Introduction: Chronic thromboembolic pulmonary hypertension (CTEPH) is one of the leading causes of pulmonary hypertension. Diagnosis of CTEPH can be established using various imaging techniques, including ventilation-perfusion scintigraphy (VQ) and multidetector computed tomography pulmonary angiography (CTPA). The aim of this study was to determine the frequency of direct pulmonary vascular, parenchymal lung, and cardiac abnormalities on CTPA in patients with CTEPH and to compare the diagnostic accuracy of both VQ scan CTPA in detecting CTEPH. Methods: We retrospectively included 54 patients who had been referred for pulmonary hypertension service (20 males, 34 females). All patients had VQ scan and CTPA within 15 days and underwent pulmonary artery endarterectomy (PEA) thereafter. VQ scans were reported according to modified PIOPED (Prospective Investigation of Pulmonary Embolism Diagnosis) criteria. CTPA was considered as diagnostic for CTEPH if it showed presence of thrombus, webs, stenosis, or perfusion lung abnormalities.

Results: The mean age of the study population was $41 \pm 10$ years. The mean pulmonary artery pressure was $53 \pm 13$ $\mathrm{mmHg}$. Fifty-three out of 54 patients in the study population had high probability VQ scan and one patient had intermediate probability. CTPA was suggestive of CTEPH in all patients. The most frequent CTPA findings in the central pulmonary arteries and peripheral arteries were presence of thrombotic materials, abnormal vessel tapering and abrupt vessels-cut off ( $76 \%$ vs $65 \%, 67 \%$ vs $48 \%$, and $48 \%$ vs $22 \%)$, respectively. The mosaic lung perfusion was present in $78 \%$ of the patients, and various cardiac morphology abnormalities were present and most common was abnormal right to left ventricle ratio $(69 \%)$.

Conclusion: Our findings indicate that both VQ scan and CTPA are highly sensitive for the detection of CTEPH confirmed by PEA. Most CTEPH patients had several pulmonary vascular, parenchymal lung and cardiac abnormalities. There was no sign with $100 \%$ sensitivity on CTPA for CTEPH detection.

Key words: Chronic thromboembolic pulmonary hypertension; VQ scintigraphy; multidetector computed tomography (CT) pulmonary angiography (CTPA); pulmonary hypertension.

Correspondence: Ahmed Fathala, MD, King Faisal Specialist Hospital and Research Center, Medical Imaging Service/Department of Radiology, MBC\#28, P.O. Box 3354, Riyadh, Saudi Arabia.

Tel.+966.552532402 - Fax: +966.144224841. E-mail: ahm35799@hotmail.com

Contributions: Both authors contributed equally to this work and approved the final version of this article for publication.

Conflict of interest: The authors declare that they have no competing interests, and all authors confirm accuracy.

Ethics approval: The study has been approved by Local Hospital Committee and informed consent was waived per our Ethics Committee for retrospective study.

Funding: No funding was received for this study.

Availability of data and materials: Individuals interested in the viewing the raw data analyzed in this manuscript are encouraged to contact the corresponding author.

Consent for publication: Not applicable. 


\section{Introduction}

Chronic thromboembolic pulmonary hypertension (CTEPH) is one of the leading causes - and the only potentially curable cause - of pulmonary hypertension. It is defined as resting mean pulmonary artery pressure of $25 \mathrm{mmHg}$ or more determined at right heart catheterization with a persistent pulmonary perfusion defect after single or repeated pulmonary embolization $[1,2]$. The pathogenesis of CTEPH is still unclear. Pulmonary emboli in patients with CTEPH do not resolve completely; instead, they follow an aberrant path of organization and recanalization, leading to characteristic abnormalities, such as luminal webs and bands, and partial or complete occlusion of pulmonary arteries $[3,4]$. Data from international prospective registry showed that $75 \%$ of patients with newly diagnosed CTEPH had history of acute pulmonary embolism and $56 \%$ had history of deep vein thrombosis [5]. In addition, in situ thrombosis and small vessel arteriopathy may play a role in the development of CTEPH [6].

Underdiagnoses of CTEPH is common for a multitude of reasons, including nonspecific symptoms, nonuniformity in diagnostics despite international guidelines, an ill-informed imaging community, and delays in referrals to expert centers [7]. Imaging plays a central role in establishing diagnoses, identifying the best candidates for pulmonary endarterectomies (PEAs), and providing post-surgical monitoring. Diagnosis of CTEPH can be established using several imaging techniques, including ventilation-perfusion scintigraphy (VQ), multidetector computed tomography (CT) pulmonary angiography (CTPA), high-resolution CT (HRCT), pulmonary subtraction angiography (DSA), and more recently magnetic resonance angiography $[8,9]$.

VQ scan has a higher sensitivity than CTPA in detecting CTEPH [10]. Multiple segmental perfusion defects that are unmatched to ventilation defects on ventilation images make CTEPH most likely diagnosis, although other rare pulmonary disorders such as veno-occlusive disease may result in the same appearance [11]. However, VQ scan does not allow determination of the location, the magnitude, and the extent of the disease and therefore, cannot predict its surgical operability. The introduction of CTPA has changed the role of VQ scan in diagnosing acute pulmonary embolism (PE). It has become the first step in the imaging method when investigating patients with suspected acute PE. The CTPA imaging features in patients CTEPH has been described in several previous reviews [12-14]. However, currently, there are few publications in the literature that investigated the presence and frequencies of CTEPH-related signs and abnormalities on CTPA, but, most of these studies included a small number of patients $[10,15,16]$.

This study was undertaken to evaluate the diagnostic accuracy of VQ scan and CTPA and the frequencies of direct pulmonary vascular, cardiac and parenchymal lung abnormalities in patients with documented chronic PE who had a positive VQ scan and underwent pulmonary artery endarterectomy (PEA) as a definitive surgical treatment for CTEPH. Specifically, we aimed to determine the frequencies of direct pulmonary vascular abnormalities such as presence of walladherent emboli and total vessel occlusion or stenosis in both central and peripheral pulmonary arteries. In addition, we aimed to determine other indirect abnormalities on CTPA such as pulmonary parenchymal abnormalities and cardiac morphology abnormalities.

\section{Materials and Methods}

\section{Patients}

This study was a single-center retrospective observational study approved by the hospital ethics committee, based on our institutional policy and IRB approval, and no informed patient's consent was needed for such a publication. It was conducted in a tertiary care center between June 2015 and June 2019 with 54 patients aged 18 years and older, who had CTEPH documented by high probability ventilationperfusion scintigraphy (VQ) scan, except one patient who had intermediate probability VQ scan. All patients underwent CTPA within 15 days from VQ scan. Subsequently, all patients underwent pulmonary artery endarterectomies (PEAs) thereafter as a definitive therapy for CTEPH. The mean time of the PEA after VQ scan was $4 \pm 3$ months.

\section{Ventilation/perfusion VQ scintigraphy}

VQ scan was performed using ${ }^{99 \mathrm{~m}} \mathrm{Tc}-$ labeled macroaggregated albumin $\left({ }^{99 \mathrm{~m}} \mathrm{TC}-\mathrm{MAA}\right)$ and ${ }^{81 \mathrm{~m}} \mathrm{Kr}$ gas $\left({ }^{81} \mathrm{Kr}\right)$. Ventilation images were acquired first and followed by perfusion images immediately. Images were acquired in four standard projections (anterior, posterior, right posterior oblique and left posterior oblique) with a medium-energy general-purpose collimator. The perfusion images were acquired after intravenous administration of $100 \mathrm{MBq}$ of ${ }^{99} \mathrm{Tc}-\mathrm{MAA}$ with the patient in supine position, an energy window of $20 \%{ }^{99} \mathrm{TC}(140 \mathrm{keV})$ and matrix of $128 \times 128$ pixels was used and approximately 400,00 counts accumulated per view. Ventilation images using ${ }^{81} \mathrm{Kr}$ were acquired in the same standard views, where ${ }^{81} \mathrm{Kr}$ was inhaled through a mouthpiece. The acquisition parameters were the same as used for perfusion study, but the $20 \%$ window was centered on the energy peak of ${ }^{81 \mathrm{~m}} \mathrm{Kr}$ (190 keV), all VQ scan studies were analyzed in consensus by two experienced nuclear medicine physicians who were unaware of CTPA results according to the modified PIOPED criteria [17]. Figure 1 showed typical high probability VQ scan.

\section{Multidetector CTPA}

CTPA was acquired using multidetector row CT scanners with light speed 16 row- detectors and a high definition 64 row-detector (GE health care) using $8 \times 12 \mathrm{~mm}$ collimation and $2.5 \mathrm{~mm}$ reconstruction. A bolus of $100 \mathrm{ml}$ nonionic iodinated contrast medium $(300 \mathrm{mg} / \mathrm{ml})$ was administrated at $4 \mathrm{ml} / \mathrm{s}$ via peripheral cannula with a variable start time that was determined using atomic bolus trigger software with a region of interest positioned at the level of the main pulmonary trunk. The images were acquired in the craniocaudal direction with Z-axis coverage, and the field of view was chosen to include the entire chest. The CTPA scans were assessed in consensus by two experienced radiologists who were unaware of VQ scan results. Primary findings that were suggestive of CTEPH included visualization of the pulmonary thrombus (main, segmental, or subsegmental arteries), calcified thrombus, recanalization, focal stenosis, post-stenotic dilation, webs, secondary signs of pulmonary hypertension (such as diameter of pulmonary arteries), right and left ventricular size and parenchymal lungs changes (such as mosaic perfusion abnormalities), and presence of pulmonary scarring or infarction (Figure $2 \mathrm{~A}-\mathrm{E}$ ).

\section{Statistical analysis}

All continuous data were expressed as mean \pm mean \pm SD and all non-continuous variables were expressed as percentages. All statistical analyses were performed with SPSS statistics software (version 24.0, IBM).

\section{Results}

\section{Demographics}

The mean age of the study population was $41 \pm 10$ years. Twenty (37\%) patients were male, and $34(63 \%)$ were female. The mean pulmonary artery pressure was $53 \pm 13 \mathrm{mmHg}$ based on right 
heart catheterization. The mean size of main pulmonary artery dimeter was $33 \pm 4 \mathrm{~mm}$, and enlarged MPA $>30 \mathrm{~mm}$ was noted in $47(87 \%)$ of patients. All patients included in this study had both VQ scan and CTPA within 15 days, and majority of patient's VQ scan was performed before CTPA. The mean time of the PEA after VQ was $4 \pm 3$ months (Table 1).

\section{Ventilation/perfusion scintigraphy findings}

Fifty-three patients out of 54 in the study population had high probability VQ scans consistent with CTEPH. Only one patient had intermediate probability VQ scan and was considered positive for CTEPH. The severity of perfusion defects was quite variable, from one or two large-mismatched perfusion defects to multiple, extensive, bilateral mismatched defects. Only one patient with intermediate VQ scan probability had typical features of CTEPH with CTPA. The incidental visualization of both kidneys was noted in four patients indicating right to left shunt.

\section{Computed tomography pulmonary angiography findings}

\section{Direct pulmonary vascular abnormalities on CTPA}

The CTPA findings of the main and lobar arteries were as follow; total vessel occlusion (vessel cut-off) 26 (48\%), thrombotic materials (wall adherent-emboli) $41(76 \%)$, webs and bands 15 (28\%), abnormal vessels narrowing (tapering) 35 (65\%), and intimal (Wall) irregularities 29 (53\%). The findings of the segmental arteries were as follow: total vessel occlusion (vessel cut-off) 12 (22\%), thrombotic materials $36(67 \%)$, webs and bands 9 (17\%), abnormal vessels narrowing (tapering) 26 (48\%), and intimal (Wall) irregularities 23 (42\%) (Table 2).

\section{Parenchymal lung and cardiac abnormalities on CTPA}

The right ventricular hypertrophy was reported in 13 (24\%) patients. The mean diastolic diameter of the right ventricle (RV) was $45 \pm 8 \mathrm{~mm}$. The mean diastolic diameter of left ventricle (LV) was $35 \pm 8 \mathrm{~mm}$. An $\mathrm{RV} / \mathrm{LV}$ ratio of more than 1.1 was seen in 37 $(69 \%)$ patients, and septal bowing was seen in $29(54 \%)$ patients. (Table 3). Mosaic attenuation of the lung parenchyma resulting in geographical variation in perfusion was reported in $47(87 \%)$ patients. Other findings, such as pulmonary scarring, pulmonary infarction, bands, peripheral irregularities, and pleural effusion, were seen in $38(70 \%)$ patients (Table 3$)$.
Table 1. Baseline characteristics $(n=54)$.

\begin{tabular}{lc}
\hline Demographics & \\
Age, years, mean \pm SD & $41 \pm 10$ \\
Male, number $(\%)$ & $20(37 \%)$ \\
Female, number $(\%)$ & $34(63 \%)$ \\
Pulmonary artery pressure, mean \pm SD mm/Hg & $53 \pm 13$ \\
Main pulmonary artery (MPA) & \\
MPA size, mean \pm SD (mm) & $33 \pm 4$ \\
MPA $\geq 30$ mm, number (\%) & $47(87 \%)$ \\
Time of the PEA after VQ, mean \pm SD (months) & $4 \pm 3$ \\
VQ scan & \\
High probability & $53(98 \%)$ \\
Intermediate probability & $1(2 \%)$ \\
\hline
\end{tabular}

PEA, pulmonary artery endarterectomy; VQ, ventilation-perfusion scintigraphy.

Table 2. Direct pulmonary vascular abnormalities on computed tomography pulmonary angiography $(n=54)$.

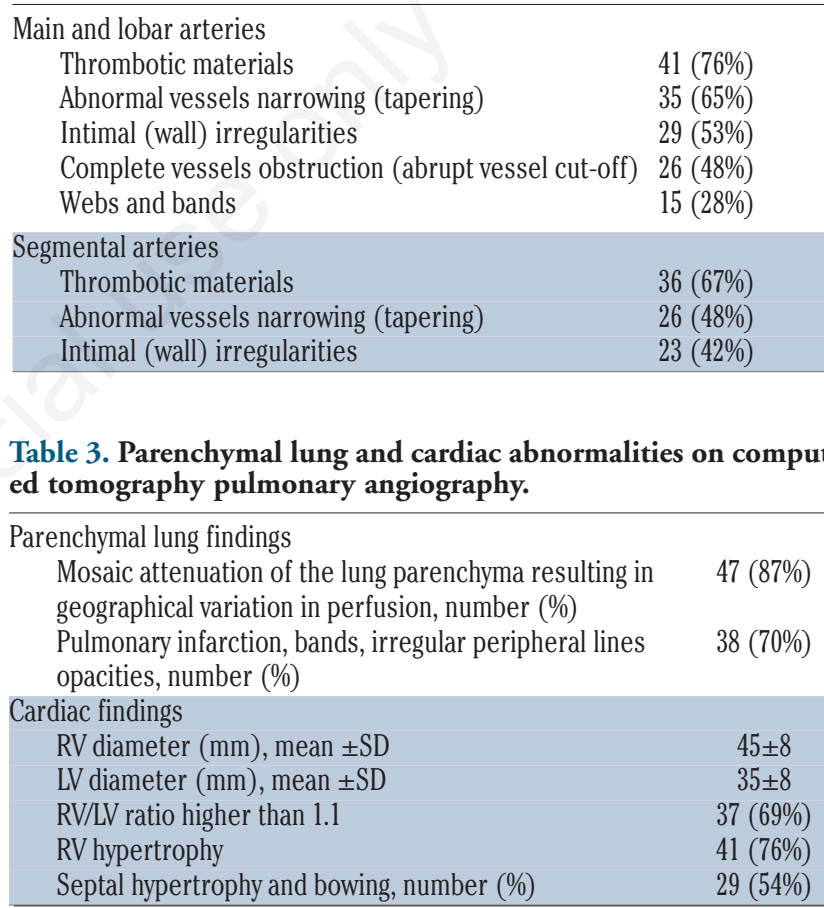

$\mathrm{RV}$, right ventricle; $\mathrm{LV}$, left ventricle.

B

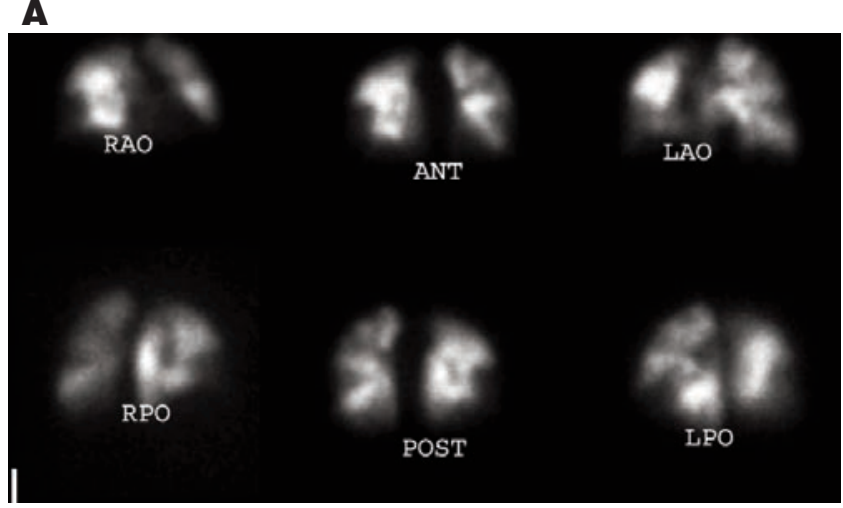

Figure 1. High probability ventilation/perfusion lung scintigraphy demonstrates extensive bilateral perfusion defects (A) consistent with CTEPH, the ventilation images were normal (B). 
A

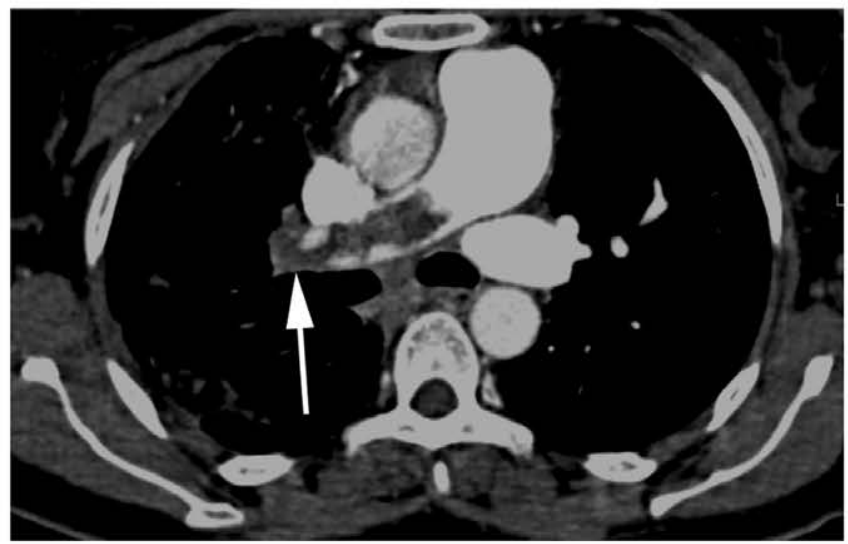

B

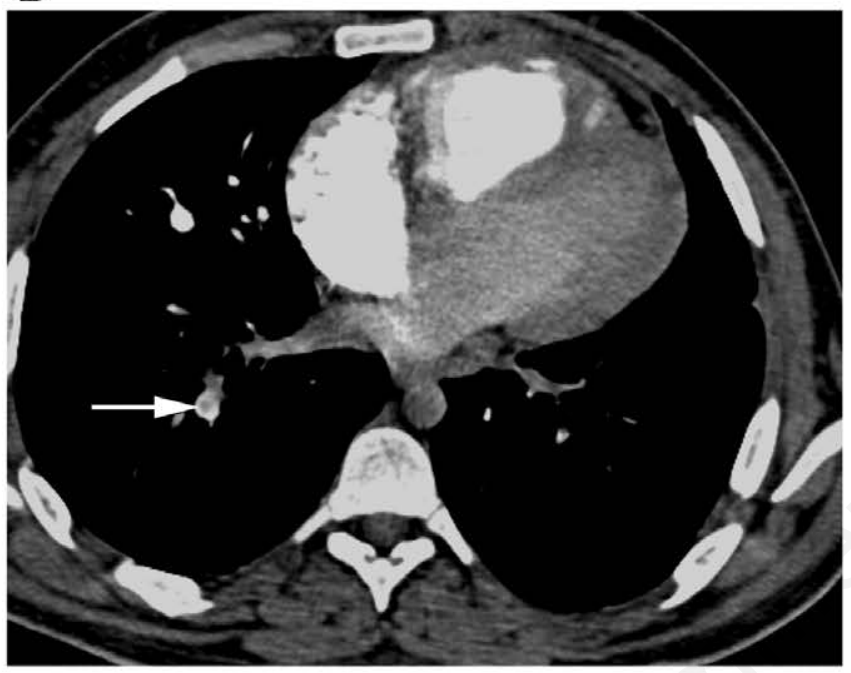

C

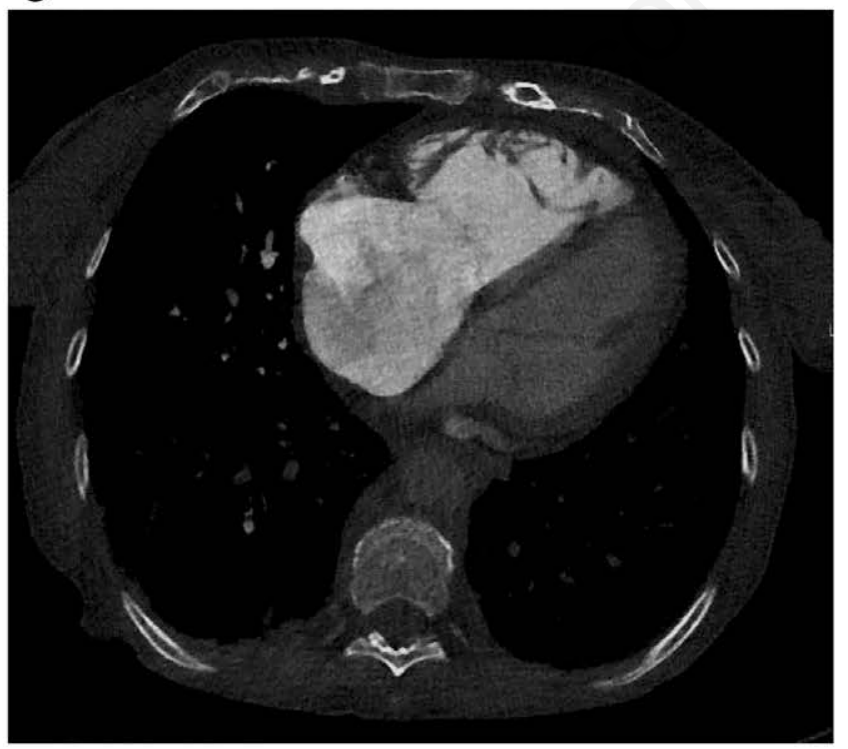

D

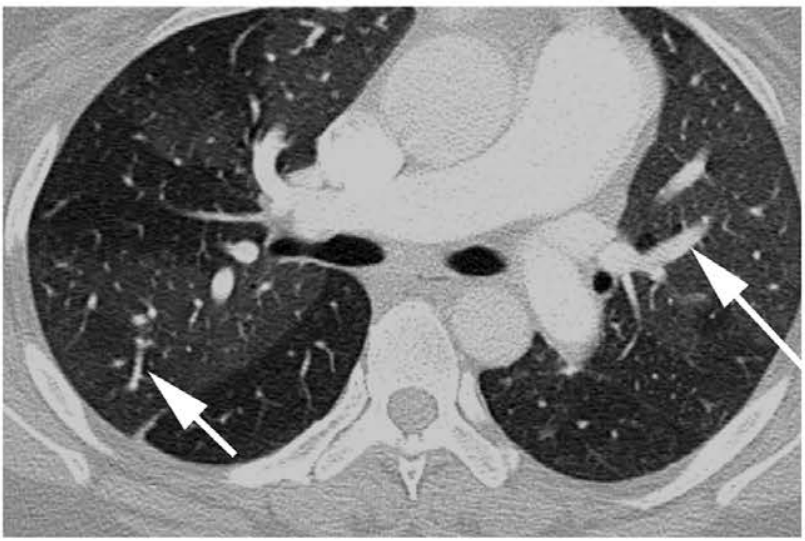

$\mathrm{E}$

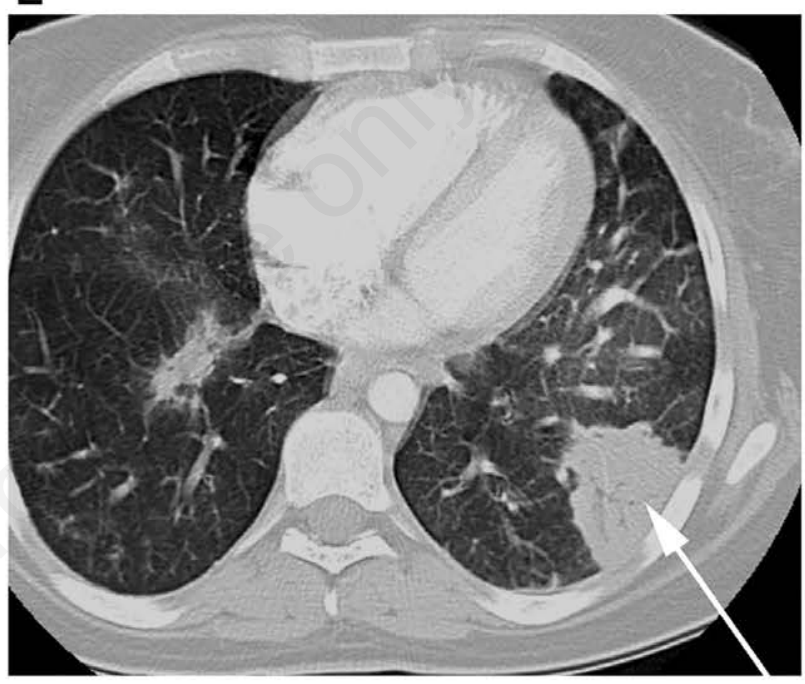

Figure 2. A) Axial image of multidetector- computed tomography pulmonary angiography (CTPA) demonstrates total obstruction of right pulmonary artery by obstructing embolus (arrow). B) Axial image of CTPA shows eccentric partial filling defect in a subsegmental artery in the right lung due to chronic embolus. C) Axial image of CTPA shows right ventricular dilation, with ratio of right ventricle to left ventricle more than $\mathbf{1 . 1}$, with septal flattening. D) Axial image of CTPA (lung window) shows a mosaic perfusion of the lung parenchyma with marked regional variation of lung parenchyma, and disparity in the size of the segmental arteries, with large diameter-vessels in regions of increased attenuation (arrows). E) Axial image of CTPA shows subpleural wedge-shaped area of consolidation, a feature indicative of pulmonary infarction. 


\section{Discussion}

In this retrospective study we determined the frequency of the direct pulmonary vessels' abnormalities, and cardiac and parenchymal lung abnormalities in patients with documented CTEPH preoperatively by VQ scan and intraoperatively. In this study PEAs were considered as the gold standard test for the presence of CTEPH, that is considered as a unique characteristic of this study compared to other previous studies. The study demonstrated presence of multiple abnormalities on CTPA with variable frequencies. The presence of wall adherent emboli, and complete vessel occlusion of the pulmonary arteries, right ventricle hypertrophy and dilatation and abnormal right ventricle to left ventricle ratio, and presence of mosaic lung perfusion were the commonest abnormalities seen on CTPA images. A previous study showed these CTPA features can be used to distinguish CTEPH from other forms of pulmonary hypertension [12]. Most importantly, there was no abnormal finding with $100 \%$ sensitivity, thus interpreting radiologists must be familiar with this observation and that the best way to establish the diagnosis of CTEPH is by using a combination of multiple abnormal findings of the pulmonary vessels, cardia morphology, and parenchymal lung changes.

Our results are comparable with prior studies that confirmed the high diagnostic accuracy of the VQ scan and CTPA in establishing CTEPH. In our study, VQ scan had a $100 \%$ and $98 \%$ sensitivity for detecting CTEPH, if both high and intermediate probability or only high probability scan was considered suggestive for CTEPH, respectively. Also, in our study, CTPA was able to detect all patients with CTEPH and final impression was consistent with presence of CTEPH that was confirmed by VQ scan preoperatively and confirmed surgically, and this yields $100 \%$ sensitivity CTPA for detecting CTEPH. This finding is concordant with a recent study that showed both VQ scan and CTPA are accurate methods for detection of CTEPH with excellent diagnostic efficacy $(100 \%$ sensitivity, 93.7\% specificity, and $96.5 \%$ accuracy of VQ scan; $96.1 \%$ sensitivity, $95.2 \%$ specificity, and 95.6 accuracy of CTPA) [18]. There must be emphasis that, CTPA sensitivity is variable from one study to another, depending on the site of the PE as well as the type of CT scanners. In an early study that used single detector computed tomography, the sensitivity of CTPA compared to pulmonary angiography was $26 \%$. Another study showed that the sensitivity of theVQ scan approaches $100 \%$ when diagnosing CTEPH [20]. It is generally accepted that normal VQ scan effectively rules out CTEPH, although, in rare circumstances, normal VQ scan with pulmonary angiographically proven PE has been reported [21]. Proposed explanations of these unusual findings involve CTEPH occurring in major vessels that are not totally obstructed, the recanalization of obstructed pulmonary vessels, and the lack of detection of small emboli in the basal segment by the VQ scan due to shine-through effects [22]. Also, it should be stressed that a low probability scan does not exclude CTEPH and does not preclude further evaluation in patients with a high suspicion of CTEPH if there is high clinical suspicion. Although high probability VQ scans are consistent with CTEPH, there are several other rare diseases that may present with large-mismatched perfusion/ventilation defects, such as vasculitis, primary pulmonary neoplasm, pulmonary veno-occlusive disease, and pulmonary atresia [22].

Although CTPA allows satisfactory visualization of all pulmonary arteries at the segmental level or higher, sub-segmental perfusion defects are difficult to visualize with CTPA. A recent metanalysis of the diagnostic accuracy of CTPA for CTEPH, which included 11 publications, resulted in a pooled sensitivity of $76 \%$. Several experts in the CTEPH field reported that, in many cases, the diagnosis of CTEPH was missed by CTPA, but unmatched perfusion defects were noted on the VQ scan [23].

Beyond pulmonary arteries evaluation, CTPA can provide additional important diagnostic findings about pulmonary parenchymal changes. In our study, a mosaic pattern was a common finding, appearing in $87 \%$ of patients. A prior study demonstrated that the evaluation of mosaic pattern areas using a high-resolution CT with minimum-intensity projection reconstruction in patients with CTEPH showed higher sensitivity than the VQ scan [24]. The mosaic pattern, characterized by sharply demarcated areas of heterogeneous attenuation in the lung parenchyma that localized to boundaries of secondary pulmonary nodules, is common in patients with CTEPH; however, it has been reported in other disease such as primary pulmonary artery hypertension and collagen vascular disease [25]. Also, dilated pulmonary artery (more than $30 \mathrm{~mm}$ or ratio of pulmonary artery to the aorta $>1$ ) is a sign of elevated pulmonary pressure and not specific for CTEPH. Other frequent findings in CTEPH include peripheral opacities caused by infarction, which are seen in $72 \%-87 \%$ of cases [26] and bronchial dilation, which is seen in as many as $64 \%$ of cases [27]. Cardiac findings (such as right ventricle dilatation), are also common findings in CTEPH patients. Right ventricle (RV) dilatation is a sign of late disease with right ventricle /left ventricle (RV/LV) diameter $>1.1$ considered to be a highly suggestive of CTEPH [28] and not significantly different among patients with central and peripheral CTEPH [29]. Leftward deviation and straightening of the interventricular septum are nonspecific signs of right ventricle pressure or volume overload [30]. Advancements in CT technologies, including dual-energy CT (DECT), ECG gated area detector CT (ADCT), cone-beam CT (CBCT), and contrast enhanced pulmonary angiography, are emerging as potential modalities for better evaluation of pulmonary vasculature and may be better for prebranch pulmonary artery angioplasty, providing greater resolution than conventional DSA, particularly in the more distal pulmonary vessels [31].

\section{Study limitations}

Our study has some limitations. First, it was a single center retrospective study with relatively small sample size, prospective multicentral studies are required to accurately determine the diagnostic accuracy of VQ scan CTPA in a larger cohort of heterogeneous patients and to determine the most reliable and reproducible futures of CTEPH on CTPA. Second, the study did not include all patients who underwent both VQ scan and CTPA; the study did include patients who underwent PEA, typically DSA is considered the gold standard test for CTEPH; however, in our study, PEA was considered the gold standard because DSA was not performed; furthermore, there was significant time variation between VQ scan and PEA.

\section{Conclusion}

In conclusion, our findings indicate that the VQ scan and CTPA are highly accurate for detecting CTEPH diagnosed by PEA. CTPA provides important complementary information to the VQ scan with regard to pulmonary parenchymal changes, pulmonary vasculature abnormalities, cardiac morphology, and CTPEA can be helpful in the diagnosis of other disorders that may produce perfusion defects on the VQ scan. A normal VQ scan effectively excludes the presence of CTEPH and may be the preferred initial diagnostic test. Abnormal VQ scan (high or intermediate probability) is accurate for detecting CTEPH. CTPA after abnormal VQ scan provides essential information regarding the extent and localization of the pulmonary emboli and accessibility for pulmonary endarterectomy. 


\section{References}

1. Pepke-Zaba J, Delcroix M, Lang I, Mayer E, Jansa P, Ambroz D, et al. Chronic thromboembolic pulmonary hypertension (CTEPH): results from an international prospective registry. Circulation 2011;124:1973-81.

2. McIntyre KM, Sasahara AA. The hemodynamic response to pulmonary embolism in patients without prior cardiopulmonary disease. Am J Cardiol 1971;28:288-94.

3. Frazier AA, Galvin JR, Franks TJ, Rosado-De-Christenson ML. From the archives of the AFIP: pulmonary vasculature: hypertension and infarction. Radiographics 2000;20:491-524; quiz 530-1, 532.

4. Dartevelle P, Fadel E, Mussot S, Chapelier A, Herve P, de Perrot $\mathrm{M}$, et al. Chronic thromboembolic pulmonary hypertension. Eur Respir J 2004;23:637-48.

5. Pengo V, Lensing AW, Prins MH, Marchiori A, Davidson BL, Tiozzo F, et al. Incidence of chronic thromboembolic pulmonary hypertension after pulmonary embolism. $\mathrm{N}$ Engl J Med 2004;350:2257-64.

6. Simonneau G, Galie N, Rubin LJ, Langleben D, Seeger W, Domenighetti $\mathrm{G}$, et al. Clinical classification of pulmonary hypertension. J Am Coll Cardiol 2004;43:5s-12s.

7. Gopalan D, Delcroix M, Held M. Diagnosis of chronic thromboembolic pulmonary hypertension. Eur Respir Rev 2017;26:160108

8. Hoeper MM, Mayer E, Simonneau G, Rubin LJ. Chronic thromboembolic pulmonary hypertension. Circulation 2006;113:201120.

9. Stein PD, Chenevert TL, Fowler SE, Goodman LR, Gottschalk A, Hales CA, et al. Gadolinium-enhanced magnetic resonance angiography for pulmonary embolism: a multicenter prospective study (PIOPED III). Ann Intern Med 2010;152:434-43, w142-3.

10. Tunariu N, Gibbs SJ, Win Z, Gin-Sing W, Graham A, Gishen P, et al. Ventilation-perfusion scintigraphy is more sensitive than multidetector CTPA in detecting chronic thromboembolic pulmonary disease as a treatable cause of pulmonary hypertension. J Nucl Med 2007;48:680-4.

11. Ryan KL, Fedullo PF, Davis GB, Vasquez TE, Moser KM. Perfusion scan findings understate the severity of angiographic and hemodynamic compromise in chronic thromboembolic pulmonary hypertension. Chest 1988;93:1180-5.

12. Grosse C, Grosse A. CT findings in diseases associated with pulmonary hypertension: a current review. Radiographics 2010;30:1753-77.

13. King MA, Ysrael M, Bergin CJ. Chronic thromboembolic pulmonary hypertension: CT findings. AJR Am J Roentgenol 1998;170:955-60.

14. Haramati A, Haramati LB. Imaging of chronic thromboembolic disease. Lung 2020;198:245-55.

15. Bergin CJ, Rios G, King MA, Belezzuoli E, Luna J, Auger WR. Accuracy of high-resolution CT in identifying chronic pulmonary thromboembolic disease. AJR Am J Roentgenol 1996;166:1371-7.

16. Remy-Jardin M, Remy J, Louvegny S, Artaud D, Deschildre F,
Duhamel A. Airway changes in chronic pulmonary embolism: CT findings in 33 patients. Radiology. 1997;203:355-60.

17. Freitas JE, Sarosi MG, Nagle CC, Yeomans ME, Freitas AE, Juni JE. Modified PIOPED criteria used in clinical practice. J Nucl Med 1995;36:1573-8.

18. He J, Fang W, Lv B, He JG, Xiong CM, Liu ZH, et al. Diagnosis of chronic thromboembolic pulmonary hypertension: comparison of ventilation/perfusion scanning and multidetector computed tomography pulmonary angiography with pulmonary angiography. Nucl Med Commun 2012;33:459-63.

19. Le Gal G, Righini M, Parent F, van Strijen M, Couturaud F. Diagnosis and management of subsegmental pulmonary embolism. J Thromb Haemost 2006;4:724-31.

20. Worsley DF, Palevsky HI, Alavi A. Ventilation-perfusion lung scanning in the evaluation of pulmonary hypertension. J Nucl Med 1994;35:793-6.

21. Alduraibi A, Fathala A. Normal ventilation/perfusion lung scan in patients with extensive chronic thromboembolism pulmonary hypertension: A case report. Radiology case reports 2019;14:5103.

22. McCann C, Gopalan D, Sheares K, Screaton N. Imaging in pulmonary hypertension, part 2: Large vessel diseases. Postgrad Med J 2012;88:317-25.

23. Robbins IM, Pugh ME, Hemnes AR. Update on chronic thromboembolic pulmonary hypertension. Trends Cardiovasc Med 2017;27:29-37.

24. Rossi A, Attina D, Borgonovi A, Buia F, De Luca F, Guidalotti PL, et al. Evaluation of mosaic pattern areas in HRCT with MinIP reconstructions in patients with pulmonary hypertension: could this evaluation replace lung perfusion scintigraphy? Eur J Radiol 2012;81:e1-6.

25. Sherrick AD, Swensen SJ, Hartman TE. Mosaic pattern of lung attenuation on CT scans: frequency among patients with pulmonary artery hypertension of different causes. AJR Am J Roentgenol 1997;169:79-82.

26. Heinrich M, Uder M, Tscholl D, Grgic A, Kramann B, Schäfers HJ. CT scan findings in chronic thromboembolic pulmonary hypertension: predictors of hemodynamic improvement after pulmonary thromboendarterectomy. Chest 2005;127:1606-13.

27. Kauczor HU, Schwickert HC, Mayer E, Schweden F, Schild HH, Thelen M. Spiral CT of bronchial arteries in chronic thromboembolism. J Comput Assist Tomogr 1994;18:855-61.

28. Chan AL, Juarez MM, Shelton DK, MacDonald T, Li CS, Lin TC, et al. Novel computed tomographic chest metrics to detect pulmonary hypertension. BMC Med Imaging 2011;11:7.

29. Ozer Gokaslan C, Onrat E, Gokaslan S. Evaluation of the CT imaging findings in the diagnosis of pulmonary hypertension due to chronic thromboembolism. Clin Respir J 2020;14:228-34.

30. Seo HS, Lee H. Assessment of right ventricular function in pulmonary hypertension with multimodality imaging. J Cardiovasc Imaging 2018;26:189-200.

31. Ogo T, Fukuda T, Tsuji A, Fukui S, Ueda J, Sanda Y, et al. Efficacy and safety of balloon pulmonary angioplasty for chronic thromboembolic pulmonary hypertension guided by cone-beam computed tomography and electrocardiogram-gated area detector computed tomography. Eur J Radiol 2017;89:270-6.

Received for publication: 9 February 2021. Accepted for publication: 31 May 2021.

This work is licensed under a Creative Commons Attribution-NonCommercial 4.0 International License (CC BY-NC 4.0).

(C) Copyright: the Author(s), 2021

Licensee PAGEPress, Italy

Multidisciplinary Respiratory Medicine 2021; $16: 753$

doi:10.4081/mrm.2021.753 